\title{
Oligomeric proanthocyanidins improve memory and enhance phosphorylation of vascular endothelial growth factor receptor-2 in senescence-accelerated mouse prone/8
}

\author{
Young A. Lee ${ }^{1}$, Eun Ju $\mathrm{Cho}^{2}$ and Takako Yokozawa ${ }^{1 *}$ \\ ${ }^{1}$ Institute of Natural Medicine, University of Toyama, 2630 Sugitani, Toyama 930-0194, Japan \\ ${ }^{2}$ Department of Food Science and Nutrition, Pusan National University, 30 Jangjeon-dong, Geumjeong-gu, Busan 609-735, \\ South Korea \\ (Received 1 May 2009 - Revised 6 August 2009 - Accepted 12 August 2009 - First published online 13 October 2009)
}

Senescence-accelerated mouse prone/8 (SAMP8), a murine model of accelerated senescence, shows age-related deficits in learning and memory. We investigated the effect of oligomeric proanthocyanidins (oligomers) on memory impairment using the SAMP8 model involving the oral administration of oligomers for 5 weeks. To analyse memory improvement in SAMP8, we performed Morris water maze, object location and object recognition tests. The oral administration of oligomers improved spatial and object recognition impairment in SAMP8. Expressions of phosphorylated neurofilament-H (P-NF-H, axon marker), microtubule-associated proteins (MAP) 2a and 2b (MAP2; dendrite marker) and synaptophysin were increased in the brains of SAMP8-administered oligomers. In particular, the expression of P-NF-H was significantly elevated in the hippocampal CA1. This indicates that oligomers result in an increase in the densities of axons, dendrites and synapses. To investigate the protective mechanisms of oligomers against brain dysfunction with ageing, we carried out a receptor tyrosine kinase phosphorylation antibody array, and clarified that the administration of oligomers led to an increase in the phosphorylation of vascular endothelial growth factor receptor (VEGFR)-2, suggesting the neuroprotective role of oligomers. The phosphorylation of VEGFR-2 was more greatly increased in the hypothalamus and choroid plexus than in other brain regions of SAMP8. Memory in oligomer-treated mice was impaired by SU1498, a VEGFR-2-specific antagonist. Elucidating the relationship between memory impairment with ageing and VEGFR-2 signalling may provide new suggestions for protection against memory deficit in the ageing brain.

Senescence-accelerated mouse prone/8: Oligomers: Memory: Vascular endothelial growth factor receptor-2: Phosphorylation: Neuroprotection

The senescence-accelerated mouse (SAM), a murine model of accelerated senescence, was developed by Takeda et al. ${ }^{(1)}$. The phenotype shows a shortened life span with an acceleration of several pathological conditions such as osteoporosis (P6), degenerative joint disease (P3), cataract (P9), hyperinflammation of the lungs (P1) and hearing impairment $(\mathrm{P} 1)^{(2)}$. One of these strains, SAM prone/8 (SAMP8), was found to exhibit age-related deficits in learning and memory. Numerous age-dependent alterations have been identified in the brain of SAMP8, such as cortical atrophy in the pyriform cortex, increased axonal dystrophy in the gracile nucleus, spongiform degeneration in the brainstem reticular formation, periodic acid Schiff-positive granular structures in the hippocampus, $\mathrm{A} 4$ protein-like immunoreactive granular structures in various regions and blood-brain barrier dysfunction ${ }^{(3)}$. There is increasing evidence that SAMP8 shows a similar pathology to the human brain in Alzheimer's disease as well as normal ageing ${ }^{(2,4)}$. On the other hand, several studies have suggested agents or methods to attenuate memory impairment with ageing, such as acetyl-L-carnitine ${ }^{(5)}$, $\alpha$-lipoic $\operatorname{acid}^{(6)}$ and caloric restriction ${ }^{(7)}$. Anti-oxidative activity limited in the cerebral cortex was suggested as the underlying mechanism of those treatments ${ }^{(8)}$. Considering that neuronal degeneration occurs in various brain regions of SAMP8, the protective role of promising agents against antiageing activity has to be investigated in various regions of the brain. Therefore, in the present study, we investigated the effect of the oligomeric form of proanthocyanidins on memory deficit in SAMP8, and the underlying mechanism in all brain regions was also observed.

Proanthocyanidins are known as condensed tannins, members of a specific group of polyphenolic compounds, and they have been reported to exhibit powerful anti-oxidant activity $^{(9,10)}$. Although proanthocyanidin is the most abundant dietary polyphenol, its high-level polymerisation results in limited absorption in vivo ${ }^{(11)}$. We previously isolated oligomeric proanthocyanidins from persimmon peel, which is usually discarded even though it is rich in phenolic

Abbreviations: BW, body weight; EC, epicatechin; EGC, epigallocatechin; EGCg, EGC 3-O-gallate; MAP2, microtubule-associated protein 2; P-NF-H, phosphorylated neurofilament-H; P-VEGFR-2, phosphorylated vascular endothelial growth factor receptor-2; SAMP8, senescence-accelerated mouse prone/8; SAMR1, senescence-accelerated mouse-resistance/1.

* Corresponding author: Dr Takako Yokozawa, fax +81 76434 5068, email yokozawa@inm.u-toyama.ac.jp 
compounds $^{(12)}$. The amount of proanthocyanidin in the peel is higher than in whole fruits. It was reported that oligomeric proanthocyanidins (oligomers) isolated from persimmon peel increased the expression of SIRT1, which is recognised as an essential factor in lifespan extension, in an $\mathrm{H}_{2} \mathrm{O}_{2}$-induced cellular senescence model ${ }^{(13)}$. Oligomer treatment also decreased the expression level of 8-hydroxy$2^{\prime}$-deoxyguanosine, a marker of oxidation in the model. In the present study, we hypothesised that the oligomeric form of proanthocyanidins would exert a beneficial effect on memory dysfunction and neuroprotection in the aged brain. Using the SAMP8 model, we investigated the effect of oligomers on spatial and object recognition memory, and the densities of axons, dendrites and synapses were observed. Furthermore, to evaluate the neuroprotective effect, vascular endothelial growth factor receptor (VEGFR)-2 and its phosphorylation were also investigated.

\section{Materials and methods}

\section{Fractionation of oligomers from proanthocyanidins of persimmon peel}

As described previously ${ }^{(14)}$, a mixture of freshly crushed persimmon peel (green peel, $5-7 \mathrm{~cm}$ in diameter, $3 \mathrm{~kg}$ ) and dried green tea leaves $(450 \mathrm{~g})$ in water containing citric acid $(240 \mathrm{~g})$ was boiled for $3 \mathrm{~h}$. At this stage, nucleophilic substitution at the C-4 positions of polymeric proanthocyanidins with monomeric tea catechins occurred, and the polymeric molecules were consequently converted into oligomers. After cooling, the insoluble materials were removed by filtration, and the filtrate was directly applied to a Sepabeads SP 825 column $(10 \mathrm{~cm}$ internal diameter $\times 45 \mathrm{~cm}$; Mitsubishi Chemical Co., Minato-ku, Tokyo, Japan). Elution with water (4 litre) washed out non-phenolic compounds consisting of citric acid, sugars, minerals, amino acids, etc. Further elution with water containing increasing amounts of ethanol (20-80\% ethanol, $20 \%$ stepwise elution, each 2 litre) yielded a mixture of oligomeric proanthocyanidin and tea catechins $(72 \cdot 2 \mathrm{~g})$. The mixture was subsequently subjected to Sephadex LH-20 column chromatography with ethanol. The monomeric tea catechins were eluted out with ethanol, and further elution with $50 \%$ aqueous acetone yielded oligomers $(51.5 \mathrm{~g})$. The degree of oligomeric polymerisation was estimated as 3.3 by quantitative HPLC analysis of thiol degradation products ${ }^{(15)}$, while the unit ratios of epigallocatechin (EGC), epicatechin (EC), EGC 3-O-gallate (EGCg) and EC 3-O-gallate in oligomers were determined as $47,15,31$ and $6 \%$, respectively.

\section{Animals and experimental protocols}

All surgical and experimental procedures were performed in accordance with the recommendations found in the Guide for the Care and Use of Laboratory Animals ${ }^{(16)}$, and approved by the Institutional Animal Care and Use Committee of the University of Toyama. Male SAMP8 (5 weeks old, Japan SLC, Shizuoka, Japan) was housed with free access to food and water, and maintained in a controlled environment $\left(22 \pm 2^{\circ} \mathrm{C}, 50 \pm 5 \%\right.$ humidity, 12 -h light cycle starting at 07.00 hours). Animals with any gross defects (tumours outside of the trunk, motor dysfunction and overt blindness) were excluded before starting the experiment. At 38 weeks old, SAMP8 was divided into three groups based on the body weight (BW). The vehicle group was given water $(n 7)$, while the other two groups were administered oligomers orally at doses of 10 and $50 \mathrm{mg} / \mathrm{kg} \mathrm{BW} / \mathrm{d}(n \mathrm{7})$ for 5 weeks using a stomach tube. Senescence-accelerated mouse-resistance/1 (SAMR1; 38 weeks old, $n$ 7) was used as a control group. At 59 weeks old, SAMP8 was divided into two groups by $\mathrm{BW}$. The vehicle group was given water (n 3), while the other group was administered oligomers orally at a dose of $50 \mathrm{mg} / \mathrm{kg} \mathrm{BW} / \mathrm{d}$ (n 3) for $7 \mathrm{~d}$ using a stomach tube. Behavioural tests were started for 2 weeks after oligomer administration. The oligomers were administered consecutively during 3 weeks of behavioural tests.

Male ddY mice (6 weeks old, Japan SLC) were housed with free access to food and water, and maintained in the controlled environment described previously. In the first experiment, the vehicle group ( $n$ 5) was injected intracerebroventricularly with $0.9 \% \mathrm{NaCl}$. VEGF-165 and VEGF-E were injected intracerebroventricularly at doses of 25 and $50 \mathrm{ng} / \mu \mathrm{l}$ (Acris antibodies $\mathrm{GmbH}$ Hiddenhausen, Germany, $n$ 5; solution: $0.9 \% \mathrm{NaCl}$ ). In the second experiment, the vehicle group ( $n$ 5) was injected intracerebroventricularly with $5 \%$ dimethyl sulphoxide in $0.9 \% \mathrm{NaCl}$. The vehicle-treated group was injected intracerebroventricularly with SU1498 (LC Laboratories, Woburn, MA, USA, $25 \mathrm{nmol} / \mu \mathrm{l}$; solution: $5 \%$ DMSO in $0.9 \% \mathrm{NaCl})$. VEGF-165 $(25 \mathrm{ng} / \mu \mathrm{l}$; solution: $0.9 \% \mathrm{NaCl})$ or VEGF-E $(25 \mathrm{ng} / \mu \mathrm{l}$; solution: $0.9 \% \mathrm{NaCl})$ was injected intracerebroventricularly with SU1498 $(25 \mathrm{nmol} / \mu \mathrm{l}$; solution: $5 \%$ DMSO in $0.9 \% \mathrm{NaCl}$ ). In the third experiment, oligomers were administered orally at a dose of $50 \mathrm{mg} / \mathrm{kg} \mathrm{BW} / \mathrm{d}(n 4)$ for $7 \mathrm{~d}$. Then, the vehicle ( $5 \%$ DMSO in $0.9 \% \mathrm{NaCl}$ ) was injected intracerebroventricularly at $60 \mathrm{~min}$ after the final administration of oligomers. Five days after vehicle injection, SU1498 $(5 \mathrm{nmol} / \mu \mathrm{l}$; solution: $5 \%$ DMSO in $0.9 \% \mathrm{NaCl})$ was injected intracerebroventricularly at $60 \mathrm{~min}$ after the final administration of oligomers. Behavioural tests were started from $3 \mathrm{~d}$ after intracerebroventricular injection.

\section{Object location test}

The object location test assessed the ability of mice to recognise the novel spatial arrangement of familiar objects ${ }^{(17)}$. For two consecutive days, mice were individually habituated to a square field box made of polyvinyl chloride $(33 \mathrm{~cm} \times 28 \mathrm{~cm}$; height $26.5 \mathrm{~cm})$ for $10 \mathrm{~min}$. Vertical white strips were put on one side of the black walls of the field, while white circles were put on the opposite wall. Two objects (plastic bottles) were placed at a fixed distance within the square field. A mouse was then placed at the opposite edge of the field, and the number of explorations of each object was recorded during a 10-min period (training session). Mice were then placed back into the same field for $24 \mathrm{~h}$ after the training session, in which one of the objects used during the training session was moved to another position in front of different visual stimuli. The mice were then allowed to explore freely for $10 \mathrm{~min}$, and the number of explorations of each object was recorded (test session). An action was scored as an exploration when a mouse touched the object with its nose or forelimbs. Mice did not step onto the bottle, and the duration of each exploratory behaviour was very 
short; therefore, the number of times contacting an object (contact times) mostly reflected the time spent exploring it, and the contact times exclude artificial exploratory behaviour such as staying near an object. A preference index, calculated as the number of times a mouse made contact with any one of the objects (training session) or the moved object (test session)
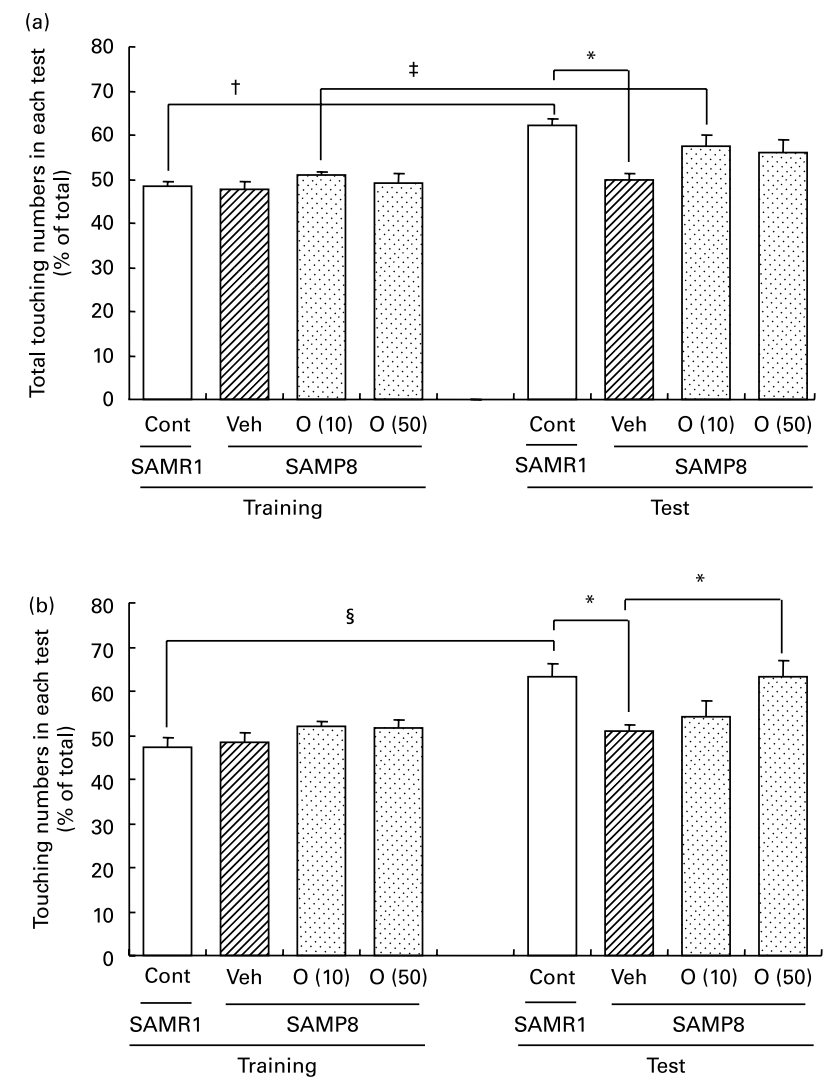

Fig. 1. Effects of oligomers $(O)$ on memory deficit in senescence-accelerated mouse prone/8 (SAMP8). (a) Thirty-eight-week-old SAMP8 was administered vehicle (Veh, water post-oral (p.o.), $n$ 7) or O ((10), $10 \mathrm{mg} / \mathrm{kg}$ body weight (BW)/d, p.o., $n 7$ or $\mathrm{O}$ (50), $50 \mathrm{mg} / \mathrm{kg} \mathrm{BW/d,} \mathrm{p.o.,} n$ 7). Age-matched senescence-accelerated mouse-resistance/1 was used as a control (Cont, $n$ 7). After $28 \mathrm{~d}$ of administration, an object location test was performed. A mouse was placed in the field, and the number of explorations of each object was recorded for $10 \mathrm{~min}$ (training session). Mice were placed back into the same field $24 \mathrm{~h}$ after the training session, in which one of the two objects used during the training session was moved to a different position in front of different scenery. The mice were then allowed to explore the area freely for $10 \mathrm{~min}$, and the number of explorations of each object was recorded (test session). The preference index was defined as the number of times a mouse made contact with any one of the objects (training session) or the moved object (test session) out of the total number of times the mouse contacted both objects (\%). ${ }^{*} P<0.05 \mathrm{v}$. Veh (one-way ANOVA followed by Bonferroni's post hoc test); ${ }^{\dagger} P=0.0005 \mathrm{v}$. Cont in training session; ${ }^{\ddagger} P=0.0213 \mathrm{v}$. $\mathrm{O}$ (10) in training session (paired $t$ test). (b) After $23 \mathrm{~d}$ of administration, a novel object recognition test was performed. A mouse was placed in the field, and the number of times it made contact with the two objects was recorded for $10 \mathrm{~min}$ (training session). Mice were placed back into the same field $24 \mathrm{~h}$ after the training session, in which one of the familiar objects used during the training session was replaced with a novel object. The mice were then allowed to explore the area freely for $10 \mathrm{~min}$, and the amount of time spent exploring each object was recorded (test session). The preference index was defined as the number of times a mouse made contact with any one of the objects (training session) or the novel object (test session) out of the total number of times the mouse contacted both objects (\%). ${ }^{*} P<0.05 v$. Veh on test trial (one-way ANOVA followed by Bonferroni's post hoc test); ${ }^{\S} P=0.0014 \mathrm{v}$. Cont in training trial (paired $t$ test). out of the total number of times it contacted both objects, was used to measure cognitive function.

\section{Novel object recognition test}

The novel object recognition test is a method to study learning and memory in mice using their tendency to interact more with a novel object than with a familiar one ${ }^{(18)}$. Two identical objects (plastic bottles) were placed at a fixed distance within a square field $(33 \times 28 \mathrm{~cm}$; height $26 \cdot 5 \mathrm{~cm})$. A mouse was then placed at the opposite edge of the field, and the number of explorations of each object was recorded during a 10-min period (training session). Mice were then placed back into the same field for $24 \mathrm{~h}$ after the training session, in which one of the familiar objects used during the training session was replaced with a novel object (a black vase). The mice were then allowed to explore freely for $10 \mathrm{~min}$, and the number of explorations of each object was recorded (test session). The number of explorations and contact times were defined and recorded in the same way as for the object location test. A preference index, calculated as the number of times a mouse made contact with any one of the objects (training session) or the novel object (test session) out of the total number of times it contacted both objects, was used to measure cognitive function.

\section{Immunoblotting}

At $1 \mathrm{~h}$ after the oral administration of oligomers to 59-weekold SAMP8, the mice were decapitated. The brains were rapidly removed from the skull, snap frozen in liquid $\mathrm{N}_{2}$ and stored at $-80^{\circ} \mathrm{C}$ until analysis. A human receptor tyrosine kinase phosphorylation antibody array (RayBiotech, Inc., Norcross, GA, USA) was performed according to the manufacturer's protocol. Brain lysates at a protein concentration of $500 \mu \mathrm{g} / \mathrm{ml}$ were incubated with array membranes for $12 \mathrm{~h}$. After washing, membranes were incubated with biotin-conjugated anti-phosphotyrosine antibody for $12 \mathrm{~h}$. Membranes were incubated with horseradish peroxidaseconjugated streptavidin-mixed detection reagent and detected by chemiluminescence with LAS-1000 plus (FUJIFILM, Tokyo, Japan). Blot densities were quantified by an image analyzer ATTO densitograph (ATTO, Tokyo, Japan). In the case of western blotting, brain lysates $(20 \mu \mathrm{g})$ were electrophoresed through an $8 \%$ SDS-polyacrylamide gel. Separated proteins were transferred electrophoretically to a pure nitrocellulose membrane, blocked with $5 \%$ skim milk solution for $1 \mathrm{~h}$ and then incubated with phosphorylated VEGFR-2 (P-VEGFR-2; Cell Signaling, Beverly, MA, USA), VEGFR2 (Santa Cruz Biotechnologies, Inc., Santa Cruz, CA, USA) or $\beta$-actin (Sigma Chemical Co., St Louis, MO, USA) antibodies overnight at $4^{\circ} \mathrm{C}$. After washing the blots, they were incubated with horseradish peroxidase-conjugated goat antirabbit or goat anti-mouse IgG secondary antibody for $2 \mathrm{~h}$ at room temperature. Each antigen-antibody complex was visualised using enhanced chemiluminescent Western blotting detection reagents (Amersham Bioscience, Piscataway, NJ, USA) and detected by chemiluminescence with LAS-1000 plus. Band densities were quantified by an image analyzer ATTO densitograph and normalised by the $\beta$-actin expression level. 


\section{Immunohistochemistry}

Brains were subsequently cut into $12-\mu \mathrm{m}$ and coronal sections using a cryostat (CM3050S, Leica, Heidelberg, Germany). Slices of the whole-brain area were fixed in $4 \%$ paraformaldehyde for $30 \mathrm{~min}$ and stained with an antibody against phosphorylated neurofilament-H (P-NF-H; Clone SMI35; Sternberger Monoclonals, Lutherville, MD, USA; 1:500; an axonal marker), microtubule-associated proteins $2 \mathrm{a}$ and $2 \mathrm{~b}$ (MAP2; Chemicon, Billerica, MA, USA; 1:500; dendritic markers), synaptophysin (Clone SY38; Chemicon; 1:500; a synaptic marker), P-VEGFR-2 (1:500), VEGFR-2 (1:100) or doublecortin (Santa Cruz; 1:100). Alexa Fluor 488- and Alexa Fluor 568-conjugated goat anti-mouse IgG (Molecular Probes, Eugene, OR, USA; 1:300) were used as secondary antibodies. Fluorescent images of the brain were captured using fluorescent microscopy (AX-80, Olympus, Tokyo, Japan) at $661 \times 878 \mu \mathrm{m}$ (the hippocampus) or $320 \times 425 \mu \mathrm{m}$ (other areas). To evaluate the expression level of each protein in each region, the fluorescence intensities of immunopositive areas (after subtracting the background) in the fixed area of squares $(19 \times 19 \mu \mathrm{m}$ : the hippocampus; $32 \times 32 \mu \mathrm{m}$ : other areas) were quantified using ATTO densitography. The background intensity was determined by staining slices without each first antibody.

\section{Statistical analysis}

The results are expressed as the means with their standard errors. In the object location and recognition tests, analyses were performed using the paired $t$ test and one-way
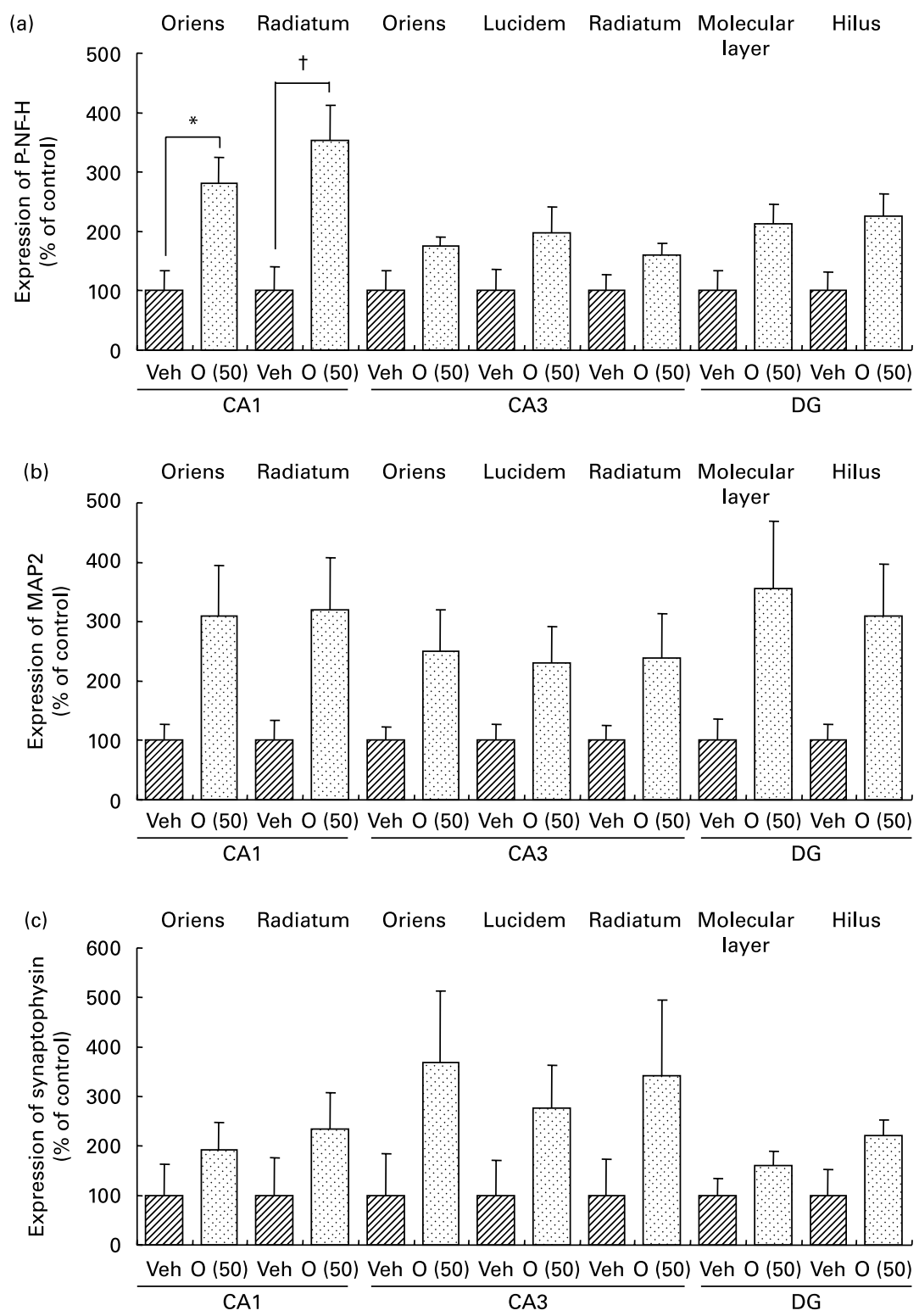

Fig. 2. Effects of oligomers on the decrease of axons, dendrites and synapses in the hippocampus. Fifty-nine-week-old senescence-accelerated mouse prone/8 was administered vehicle (Veh, water post-oral (p.o.), $n$ 3) or oligomers (O (50), $50 \mathrm{mg} / \mathrm{kg}$ body weight/d, p.o., $n 3$ ). After $7 \mathrm{~d}$ of administration, brain slices were immunostained with phosphorylated neurofilament-H (P-NF-H) (a), microtubule-associated protein 2 (MAP2) (b) and synaptophysin antibodies (c). The intensities of immunopositive areas in the hippocampus were quantified. ${ }^{*} P=0.0243 \mathrm{v} . \mathrm{O}(50) ;{ }^{\dagger} P=0.0344 \mathrm{v} . \mathrm{O}(50)$ (Student's $t$ test). 
ANOVA followed by Bonferroni's post hoc test. The immunohistochemical results were analysed by Student's $t$ test. All analyses were conducted using the statistical software Sigma Stat 1.0 for windows.

\section{Results}

Oligomers ameliorate impairment of spatial memory and object recognition in senescence-accelerated mouse prone/8

The oligomeric form of proanthocyanidin was administered orally every day for 2 weeks to SAMP8, and then an object location test was performed. Compared with the training session, SAMR1 and SAMP8 treated with oligomers $(10 \mathrm{mg} / \mathrm{kg}$ $\mathrm{BW} / \mathrm{d}$ ) showed significantly more frequent exploratory behaviours toward the moved than the fixed object (Fig. 1(a)).

Fig. 1(b) shows the effect of oligomers on object recognition memory deficits in SAMP8. Visual object recognition memory was evaluated using a novel object recognition test. SAMR1 and SAMP8 treated with oligomers $(50 \mathrm{mg} / \mathrm{kg}$ $\mathrm{BW} / \mathrm{d}$ ) showed more significant and frequent exploratory behaviours toward the novel than the familiar object in the test session.

Oligomers increase the density of dendrites and synapses in the brain of senescence-accelerated mouse prone/8

The levels of P-NF-H, MAP2 and synaptophysin in the brains of mice (59 weeks old) were measured by immunohistochemistry. The observed brain regions were the frontal cortex, seven subregions in three hippocampal regions (stratum oriens and stratum radiatum in CA1, stratum oriens, stratum lucidum and stratum radiatum in $\mathrm{CA} 3$, and molecular layer and hilus in the dentate gyrus) and the striatum. In the cerebral cortex and striatum, levels of P-NF-H, MAP2 and synaptophysin remained unchanged on the administration of the oligomeric form of proanthocyanidin (data not shown).

Fig. 2 shows the effect of oligomers on axons, dendrites and synapses in the oriens and radiatum of hippocampal CA1; oriens, lucidum and radiatum of hippocampal CA3; and molecular layers and hilius of the dendate gyrus. The expression levels of P-NF-H were increased in oligomer-administered compared with vehicle-treated mice. Levels were significantly elevated in the CA1 radiatum and oriens (Fig. 2(a)). The expression levels of MAP2 were slightly increased in oligomer-administered compared with vehicle-treated mice (Fig. 2(b)). In addition, synaptophysin was slightly increased in oligomer-administered mice, without a significant difference in comparison with vehicle-treated mice (Fig. 2(c)).

\section{Oligomers increase phosphorylation of vascular endothelial growth factor receptor-2 in the brain of senescence- accelerated mouse prone/8}

Our preliminary results using a phosphorylated receptor antibody array showed that the administration of oligomers for $7 \mathrm{~d}$ increased phosphorylation levels of VEGFR-2 in the SAMP8 brain (data not shown). The effect of oligomeric proanthocyanidins on the expressions of P-VEGFR-2 and VEGFR-2 was observed by immunoblotting (Fig. 3). The administration of oligomers resulted in an increase in the (a) Expression ratio

$1 \cdot 0$

$1 \cdot 3$

P-VEGFR-2

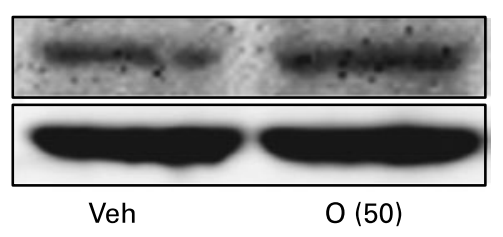

(b) Expression ratio

$1 \cdot 0$

$1 \cdot 1$

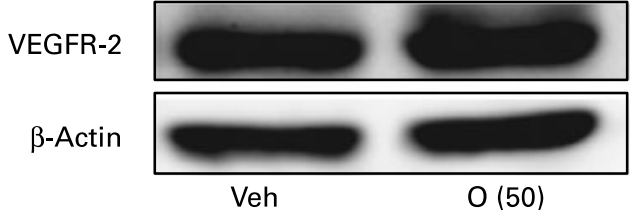

Fig. 3. Effects of oligomers on phosphorylated vascular endothelial growth factor receptor-2 (P-VEGFR-2) and VEGFR-2 expression. Fifty-nine-weekold senescence-accelerated mouse prone/8 was administered vehicle (Veh, water post-oral (p.o.), $n$ 1) or oligomers (O (50), $50 \mathrm{mg} / \mathrm{kg}$ body weight/d, p.o., $n$ 1). After $7 \mathrm{~d}$ of administration, brain lysates were immunoblotted with antibodies for P-VEGFR-2 (a) or VEGFR-2 (b). Expression intensities were divided by $\beta$-actin expressions to generate ratios.

expressions of P-VEGFR-2 and non-P-VEGFR-2 (Fig. 3(a) and (b)). In addition, the effect of oligomers on the expression in various brain regions was also investigated (Fig. 4). Brain slices of oligomer-treated SAMP8 were immunostained for the measurement of P-VEGFR-2. Increased expressions of P-VEGFR-2 were observed in the cerebral cortex, hypothalamus, choroid plexus and hippocampal CA3 layer of SAMP8 (Fig. 5(a)). In particular, the P-VEGFR-2 level in the hypothalamus of oligomer-treated SAMP8 was significantly increased compared with vehicle-treated mice. On the other hand, expression levels of VEGFR-2 in SAMP8 groups-administered oligomers did not show significant changes compared with vehicle-administered ones in all brain regions observed (Fig. 5(b)).

\section{Oligomers affected memory by a signal cascade through vascular endothelial growth factor receptor-2}

To elucidate the effect of VEGFR-2 on memory, we investigated the inhibitory effects of a VEGFR-2-specific antagonist on ligand-induced memory. Visual recognition memory was assessed using a novel object recognition test. As shown in Fig. 6, vehicle-treated mice showed significantly more frequent exploratory behaviours toward a novel object than a familiar object in the test session. However, the memory of vehicle-treated mice was impaired by SU1498 injection. In particular, mice treated with VEGF-E $(25 \mathrm{ng} / \mu \mathrm{l})$ showed a significant increase in exploratory behaviour toward a novel object compared with vehicle-treated mice, but not mice treated with VEGF-A $(25 \mathrm{ng} / \mu \mathrm{l})$. The memory enhancement induced by VEGF-E injection was markedly inhibited by SU1498. However, the memory of VEGF-165-treated mice was not impaired by SU1498. On the other hand, oligomertreated mice showed more frequent exploratory behaviours toward a novel object than a familiar one in the test session. These exploratory behaviours were inhibited by SU1498. 
(a) P-VEGFR-2
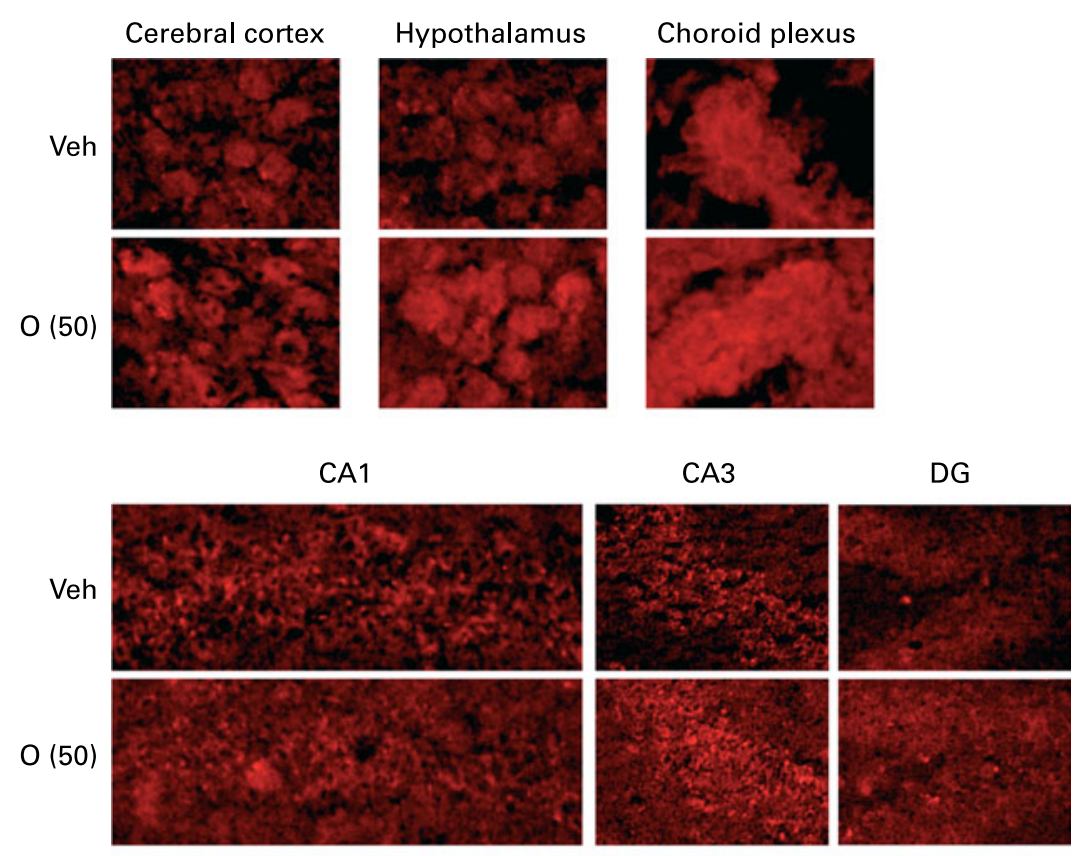

(b) VEGFR-2
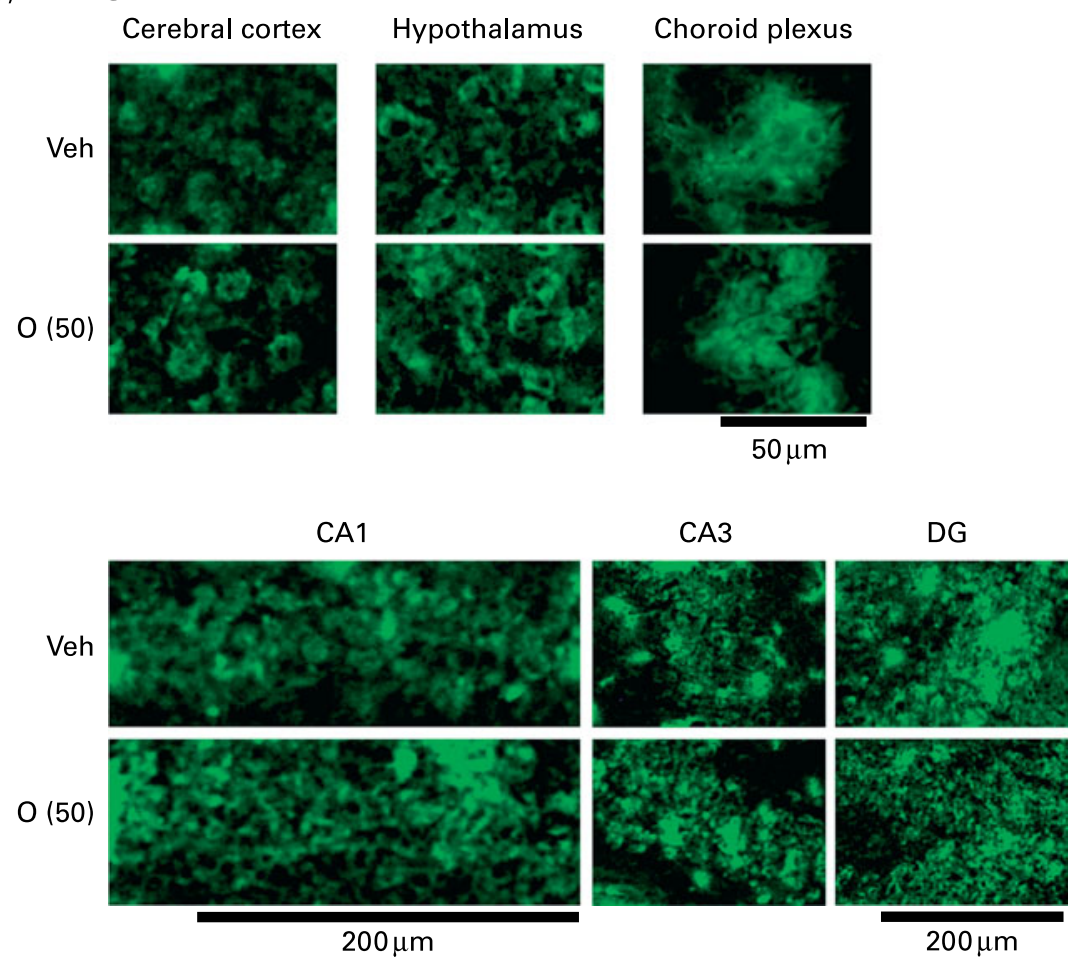

Fig. 4. Effects of oligomers on phosphorylated vascular endothelial growth factor receptor-2 (P-VEGFR-2) and VEGFR-2 expression in various brain regions. Fifty-nine-week-old senescence-accelerated mouse prone/8 was administered vehicle (Veh, water post-oral (p.o.), $n$ 3) or oligomers (O (50), $50 \mathrm{mg} / \mathrm{kg}$ body weight/d, p.o., $n$ 3). After $7 \mathrm{~d}$ of administration, brain slices were immunostained with P-VEGFR-2 (a) and VEGFR-2 (b) antibodies.

\section{Discussion}

SAMP8 developed age-related cognitive deficit at as early as 4 months and had a short lifespan relative to SAMR1. SAMP8 shows a decrease in the release of acetylcholine and noradrenaline in comparison with age-matched SAMR1 $1^{(19,20)}$.
Many age-dependent alterations in various brain regions such as the cerebral cortex and hippocampus at an early stage in SAMP8 have been suggested as causes of memory deficit $^{(21,22)}$. In the hippocampus, there was a greater increase of glial fibrillary acidic protein as an astrocyte marker in 

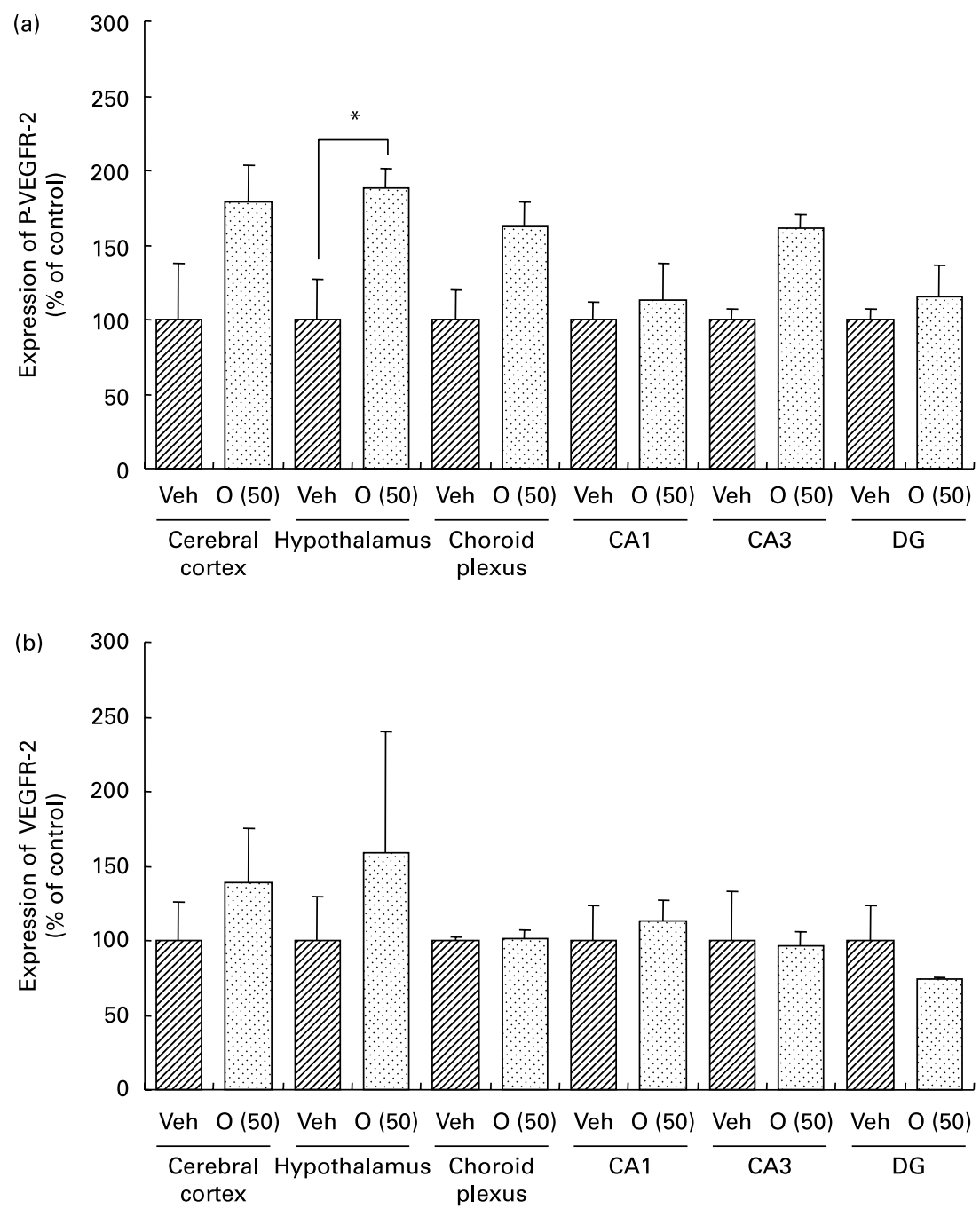

Fig. 5. Intensities of phosphorylated vascular endothelial growth factor receptor-2 (P-VEGFR-2)- (a) and VEGFR-2 (b)-positive areas were quantified in the cerebral cortex, hypothalamus and choroid plexus, and the CA1, CA3 and dentate gyrus (DG) of the hippocampus. ${ }^{*} P=0.0429 \mathrm{v}$. O (50) (Student's $t$ test).

CA1-3 regions of SAMP8 compared with age-matched SAMR1, indicating enhanced reactive gliosis in aged SAMP8 ${ }^{(23)}$. Tanaka et al. ${ }^{(24)}$ reported the severe loss of oligodendrocytes in the hippocampal CA1 of SAMP8. Moreover, neuronal loss and lower-level expressions of glial cell linederived neurotrophic factor in the hippocampal CA1 associated with memory impairment of SAMP8 were reported ${ }^{(25)}$. Therefore, hippocampal dysfunctions of SAMP8 have been considered to be a major cause of age-dependent memory impairment. Various candidate therapeutic agents for memory dysfunction in SAMP8 have been reported, such as acetyl-L-carnitine, $\alpha$-lipoic acid, Choto-san (a herbal formula medicine) and caloric restriction ${ }^{(5-7,26)}$. In those studies, oxidative stress was focused on as a cause of memory impairment in SAMP8, although the change of oxidative stress was limited in the cerebral cortex. Additionally, neuronal morphological evaluations were insufficient in those studies.

We previously reported that oligomers attenuated the expression level of 8-hydroxy-2'-deoxyguanosine as a DNA damage maker and increased the expression of SIRT1, which is recognised as an essential factor in lifespan extension in an $\mathrm{H}_{2} \mathrm{O}_{2}$-induced cellular senescence model ${ }^{(13)}$. These bioactivities were stronger in the oligomer-treated group than the group treated with non-oligomerised proanthocyanidins showing high-level polymerisation. Ageing is a progressive physiological change in an organism that leads to senescence, or a decline of biological functions, and of the organisms' ability to adapt to metabolic stress. The process increases the prevalence of learning and memory deficits. For example, in aged rats, a loss of synapses in the dentate gyrus and an alteration of $\mathrm{Ca}^{2+}$ regulation in the CA1 area lead to a decline of synaptic plasticity, resulting in a change of interactions among hippocampal networks and deficits in the storage and retrieval of information regarding the spatial organisation of the environment ${ }^{(27)}$. Therefore, we expected that the anti-ageing effect of oligomers may improve ageassociated memory impairment. In the present study, oligomers improved spatial and object recognition memory in SAMP8. The memory improvements seen in SAMP8 were almost to the same levels as those of SAMR1. To investigate the neurological changes brought about by the oral administration of oligomers, we carried out an immunohistological 


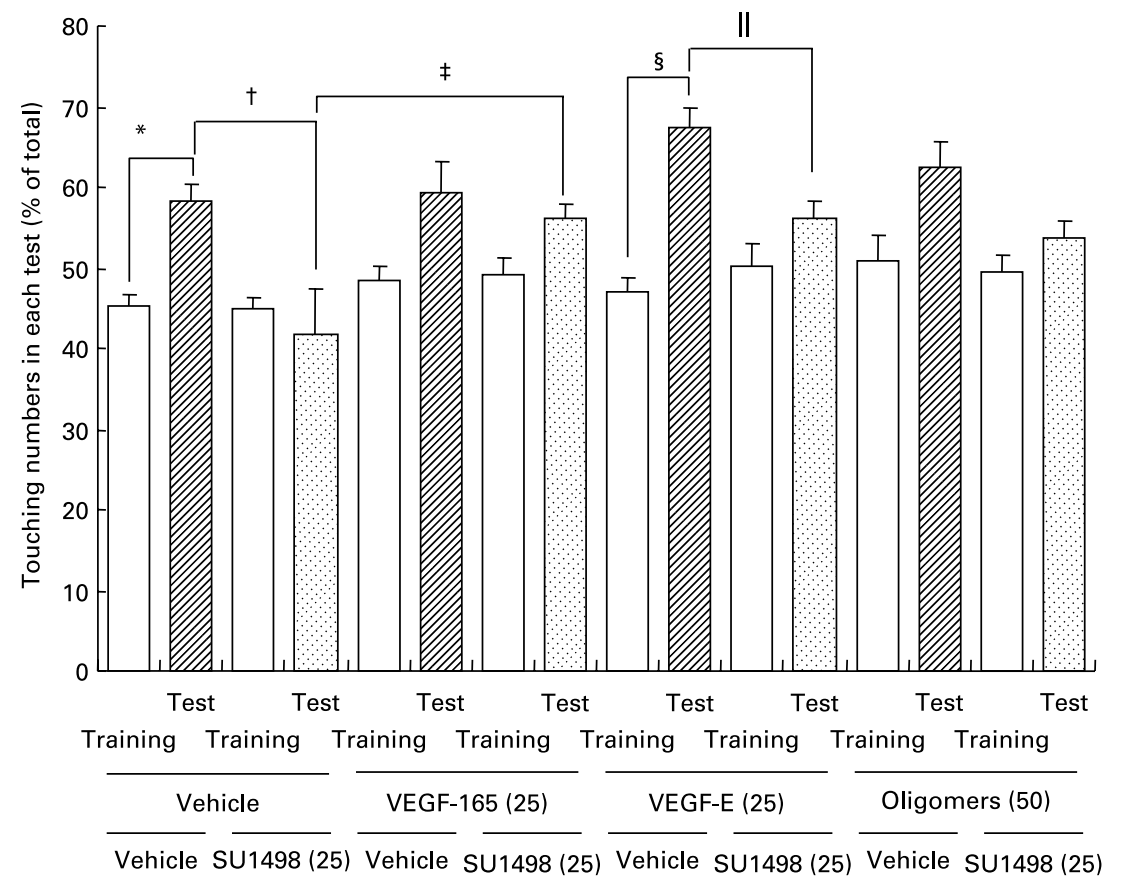

Fig. 6. Effects of oligomers on object recognition memory deficit. Six-week-old male ddY mice were injected intracerebroventricularly with vehicle $(5 \%$ dimethyl sulphoxide (DMSO) in $0.9 \% \mathrm{NaCl}, n$ 5) or SU1498 $(25 \mathrm{nmol} / 1 \mathrm{ml}$; solution: $5 \%$ DMSO in $0.9 \% \mathrm{NaCl})$. Vascular endothelial growth factor (VEGF)-165 (25 ng/ml, $n 5)$ or VEGF-E $(25 \mathrm{ng} / \mathrm{ml}, n 5)$ was injected simultaneously with vehicle or SU1498 $(25 \mathrm{nmol} / \mathrm{ml})$ intracerebroventricularly. Three days after injection, a novel object recognition test was performed. Six-week-old male ddY mice were administered oligomers orally at a dose of $50 \mathrm{mg} / \mathrm{kg}$ body weight/d $(n 4)$ for $7 \mathrm{~d}$. Then, vehicle ( $5 \% \mathrm{DMSO}$ in $0.9 \% \mathrm{NaCl}$ ) was injected intracerebroventricularly at $60 \mathrm{~min}$ after the final administration of oligomers. Five days after vehicle injection, SU1498 $(5 \mathrm{nmol} / \mathrm{ml}$; solution: $5 \% \mathrm{DMSO}$ in $0.9 \% \mathrm{NaCl})$ was injected intracerebroventricularly at $60 \mathrm{~min}$ after the final administration of oligomers. Three days after injection, a novel object recognition test was performed. A mouse was placed in the field, and the number of explorations of each object was recorded for 10 min (training session). Mice were placed back into the same field $24 \mathrm{~h}$ after the training session, in which one of the familiar objects used during the training session was replaced with a novel object. The mice were then allowed to explore the area freely for $10 \mathrm{~min}$, and the number of explorations of each object was recorded (test session). The preference index was defined as the number of times a mouse made contact with any one of the objects (training session) or the novel object (test session) out of the total number of times it contacted both objects (\%). ${ }^{\star} P=0.0002$ ( $n$ 9, paired $t$ test); ${ }^{\dagger} P=0.0371$ ( $n$ 5, Student's $t$ test); ${ }^{\ddagger} P=0.0317$ ( $n$ 5, Student's $t$ test); ${ }^{\S} P=0.0051$ ( $n$ 5, paired $t$ test); ${ }^{\prime \prime} P=0.0107$ ( $n$ 5, Student's $t$ test).

analysis in the brain of SAMP8. The oral administration of oligomers increased expression levels of P-NF-H, MAP2 and synaptophysin in the hippocampus, but this was not observed in regions of the cerebral cortex and striatum of SAMP8. In particular, the expression of P-NF-H significantly increased in the hippocampal CA1 by oligomer administration. P-NF$\mathrm{H}$ is used as a marker of axons, since the phosphorylated form of NF-H is translocated into axons ${ }^{(28)}$. In the hippocampus of aged mice, fragments of degenerated axons were also increased, although reductions of neuronal numbers are small in this region ${ }^{(29)}$. Axonal termination to the spine is a necessary step for synaptogenesis. Considering synaptic losses in the hippocampal CA1 and 3 and the parietal cortex in SAMP8 ${ }^{(30)}$, as well as in the hippocampal CA1, CA3 and dentate gyrus in aged rats ${ }^{(31)}$, axonal regeneration is important for improving hippocampal function. Therefore, the increased density of axons in the hippocampal CA1 was suggested to have a protective role against memory loss with ageing.

Previous studies have suggested that oxidative stress is a major cause of memory impairment in SAMP8. Hippocampus-specific modulation by oligomers is not explained by an anti-oxidative effect, since only the cerebral cortex is susceptible to oxidative stress in SAMP8 and not the hippocampus $^{(8)}$. Therefore, to investigate target molecules by the oral administration of oligomers in the brain of SAMP8, we performed a receptor tyrosine kinase phosphorylation antibody array, and clarified that oligomer treatment increased the phosphorylation of VEGFR-2. Expressions of VEGFR-2 were identified in the cerebral cortex, hippocampus and choroid plexus of adult as well as neonatal rodents ${ }^{(32,33)}$. However, the localisation of VEGFR-2 in the brain of SAMP8 has yet to be clarified. In the present study, VEGFR-2 expressions were also detected in the striatum and hypothalamus of SAMP8, as well as the cerebral cortex, hippocampus and choroid plexus. In neurons, stimulation by VEGFR-2 among protein tyrosine kinase receptors of VEGF is linked to Akt/PKB activation and neuronal protection in hypoxic preconditioning ${ }^{(34)}$. Moreover, VEGFR-2 mediated a protective effect through phosphatidylinositol-3-kinase/Akt- and mitogenactivated protein/extracellular signal-regulated kinase/ extracellular signal-regulated kinase-signalling pathways in glutamate-induced toxicity ${ }^{(35)}$. In particular, memory enhancement in recombinant adeno-associated viral vectors expressing human VEGF-injected mice was inhibited by the injection of dominant-negative mutant VEGFR-2 ${ }^{(36)}$. This indicates that VEGF/VEGFR-2 is associated directly with neuronal signalling. VEGF also exerts indirect effects on neurons. Moreover, the topical administration of VEGF to the surface of the brain reduces infarct size, and intraventricular VEGF enhanced the survival of newly generated neurons in the dentate gyrus and subventicular zones after focal cerebral ischaemia ${ }^{(37)}$. In the present study, we firstly showed that memory enhancement 
through oligomer treatment was inhibited by SU1498. Considering that VEGF-E-induced memory was also inhibited by SU1498, oligomers or their metabolites may regulate memory by the activation of VEGFR-2.

We elucidated that the administration of oligomers increased the phosphorylation of VEGFR-2 in the hippocampal CA3 region, suggesting that oligomeric metabolites may directly affect the hippocampus, like the VEGFR-2 ligand. It has been reported that $\mathrm{Ca}^{2+}$ influx and synaptic transmission by VEGF in the hippocampus influence the generation of long-term changes in synaptic efficacy ${ }^{(38)}$. VEGF also stimulates neurite outgrowth via Rho/Rho kinase signalling in cerebral cortical neurons ${ }^{(39)}$. Interestingly, changes in the synapes and neurites induced by VEGF are caused by the activation of VEGFR-2 rather than VEGFR-1. Therefore, we speculated that the phosphorylation of VEGFR-2 induced by the administration of oligomers within the hippocampus may be related to an increase in the densities on neurites and synapses in the hippocampus.

The administration of oligomers increased the phosphorylation of VEGFR-2 in the hypothalamus and choroid plexus as well as the hippocampus. The hypothalamus is contained in the Papez circuit. The Papez circuit is a sensory circuit involving the thalamus, sensory cortex (especially the cingulate region), hippocampus and mammillary body of the hypothalamus $^{(40)}$. It has been reported that lesions in the Papez circuit are associated with amnesia and the impairment of recognition memory ${ }^{(41)}$. Therefore, we speculate that the hypothalamus is activated by the phosphorylation of VEGFR-2, which may affect the hippocampus through the Papez circuit.

The choroid plexus is made up of numerous villi that project into the ventricles of the brain. Each villus is composed of a single layer of epithelial cells overlying a core of connective tissues and blood capillaries ${ }^{(42)}$. The choroid plexus is involved in the most basic aspects of neural function, including maintaining the extracellular milieu of the brain by actively modulating chemical exchange between the cerebrospinal fluid and brain parenchyma, surveying the chemical and immunological status of the brain, detoxifying the brain, secreting a nutritive cocktail of polypeptides and participating in repair processes following trauma. This diversity of functions may mean that even modest changes in the choroid plexus can have far-reaching effects ${ }^{(43)}$. Actually, a host of growth factors and other neuroprotective agents given via the cerebrospinal fluid can minimise the adverse effects of stroke on the rat hippocampus. Multiple functional failures including a decrease of cerebrospinal fluid as well as the atrophy of choroidal epithelial cells showed in normal ageing as well as advanced Alzheimer's disease indicate that the maintenance of cerebrospinal fluid through the choroid plexus may have beneficial effects against neurodegenerative diseases ${ }^{(44)}$. Moreover, it has been reported that the intracerebroventricular injection of nerve growth factor or insulin-like growth factor-1 improved memory deficit and hippocampal deterioration ${ }^{(45,46)}$. Therefore, we speculate that oligomers may induce the secretion of some peptides after the phosphorylation of VEGFR-2 in the choroid plexus, and then this peptide may induce changes in the hippocampus.

We previously elucidated that the oligomers consisted of various combinations of four types of monomer: EGC, EC,
EGCg and EC 3-O-gallate. Oligomers containing dimers, trimers and tetramers of EGC, EC, EGCg and EC 3-O-gallate are considered to exert a stronger activity than polymers. De Boer et al. ${ }^{(47)}$ demonstrated that EC 3-O-gallate and EGCg stimulated SIRT1 more effectively than EC and EGC, since galloyl and catechol groups are essential for the repair of DNA damage. Therefore, the structural difference between oligomers and polymers is considered to be an important factor regarding proanthocyanidin's action, and it also affects the utilisation in biological systems. It is absorbed through the gut barrier, and its absorption depends on the degree of polymerisation. Low molecular weight proanthocyanidins are known as sustained release antioxidants; on the contrary, high molecular weight proanthocyanidins can exert their anti-oxidant activity in the digestive tract and protect lipids, proteins and carbohydrates from oxidative damage during digestion and spare soluble antioxidants ${ }^{(48)}$. Furthermore, EC and EGCg are distributed in the brain by their absorption into plasma after oral injection, and these monomeric forms may act as potential therapeutic agents in neurodegenerative diseases ${ }^{(49)}$. This suggests that those monomeric forms can act as potential therapeutic agents against neurodegenerative diseases in the brain. In addition, it has been reported that dimers and trimers of proanthocyanidins can be absorbed into epithelial cells such as Caco-2 cells ${ }^{(50)}$, suggesting that oligomers may act both oligomerically and monomerically. We must elucidate the similarities and differences in activities and functional mechanisms between oligomers and metabolites including monomers in vivo.

The present study indicated that the oral administration of oligomers improved memory impairment in SAMP8. In particular, the density of axons in the hippocampal CA1 was significantly increased by oligomer administration. Moreover, the administration of oligomers increased the phosphorylation of VEGFR-2 in the hippocampal CA3, hypothalamus and choroid plexus. We speculate that memory improvement accompanied with histological changes may be induced directly in the hippocampus and indirectly in the hypothalamus and choroid plexus through VEGFR-2 signalling. In the present study, we elucidated the protective effect of oligomers against memory impairment with ageing. VEGFR-2 signalling may give us new insight into ways to protect against memory deficit in the ageing brain.

\section{Acknowledgements}

The present work was supported in part by the Grants-in-Aid (C) from the Ministry of Education, Culture, Sports, Science and Technology, Japan (no. 19500661 to T. Y.). The authors state that there are no conflicts of interest. Y. A L. and E. J. C. conducted the experimental work. T. Y. designed the experiment and wrote the manuscript.

\section{References}

1. Takeda T, Matsushita T, Kurozumi M, et al. (1997) Pathobiology of the senescence-accelerated mouse (SAM). Exp Gerontol 32, 117-127.

2. Markowska AL, Spangler EL \& Ingram DK (1998) Behavioral assessment of the senescence-accelerated mouse (SAM P8 and R1). Physiol Behav 64, 15-26. 
3. Kawamata T, Akiguchi I, Yagi H, et al. (1997) Neuropathological studies on strains of senescence-accelerated mice (SAM) with age-related deficits in learning and memory. Exp Gerontol 32, $161-169$.

4. Flood JF \& Morley JE (1998) Learning and memory in the SAMP8 mouse. Neurosci Biobehav Rev 22, 1-20.

5. Yasui F, Matsugo S, Ishibashi M, et al. (2002) Effects of chronic acetyl-L-carnitine treatment on brain lipid hydroperoxide level and passive avoidance learning in senescence-accelerated mice. Neurosci Lett 334, 177-180.

6. Farr SA, Poon HF, Dogrukol-Ak D, et al. (2003) The antioxidants alpha-lipoic acid and $N$-acetylcysteine reverse memory impairment and brain oxidative stress in aged SAMP8 mice. J Neurochem 84, 1173-1183.

7. Komatsu T, Chiba T, Yamaza H, et al. (2008) Manipulation of caloric content but not diet composition, attenuates the deficit in learning and memory of senescence-accelerated mouse strain P8. Exp Gerontol 43, 339-346.

8. Sato E, Kurokawa T, Oda N, et al. (1996) Early appearance of abnormality of microperoxisomal enzymes in the cerebral cortex of senescence-accelerated mouse. Mech Ageing Dev 92, 175-184.

9. Dixon RA, Xie DY \& Sharma SB (2005) Proanthocyanidins - a final frontier in flavonoid research? New Phytol 165, 9-28.

10. Xie DY \& Dixon RA (2005) Proanthocyanidin biosynthesis still more questions than answers? Phytochemistry 66, 2127-2144.

11. Manach C, Williamson G, Morand C, et al. (2005) Bioavailability and bioefficacy of polyphenols in humans. I. Review of 97 bioavailability studies. Am J Clin Nutr 81, 230S-242S.

12. Gorinstein S, Zachwieja Z, Folta M, et al. (2001) Comparative contents of dietary fiber, total phenolics, and minerals in persimmons and apples. J Agric Food Chem 49, 952-957.

13. Lee YA, Cho EJ \& Yokozawa T (2008) Protective effect of persimmon (Diospyros Kaki) peel proanthocyanidin against oxidative damage under $\mathrm{H}_{2} \mathrm{O}_{2}$-induced cellular senescence. Biol Pharm Bull 31, 1265-1269.

14. Lee YA, Cho EJ \& Yokozawa T (2008) Effects of proanthocyanidin preparations on hyperlipidemia and other biomarkers in mouse model of type 2 diabetes. J Agric Food Chem 56, $7781-7789$.

15. Tanaka T, Takahashi R, Kouno I, et al. (1994) Chemical evidence for the de-astringency (insolubilization of tannins) of persimmon fruit. J Chem Soc Perkin Trans I, 3013-3022.

16. National Research Council (1996) Guide for the Care and Use of Laboratory Animals. Bethesda, MD: National Institute of Health.

17. Save E, Poucet B, Foreman N, et al. (1992) Object exploration and reactions to spatial and nonspatial changes in hooded rats following damage to parietal cortex or hippocampal formation. Behav Neurosci 106, 447-456.

18. Bevins RA \& Besheer $\mathrm{J}$ (2006) Object recognition in rats and mice: a one-trial non-matching-to-sample learning task to study 'recognition memory'. Nat Protocols 1, 1306-1311.

19. Zhao XH \& Nomura Y (1990) Age-related changes in uptake and release on L- $\left[{ }^{3} \mathrm{H}\right]$ noradrenaline in brain slices of senescence accelerated mouse. Int J Dev Neurosci 8, 267-272.

20. Zhao XH, Kitamura Y \& Nomura Y (1992) Age-related changes in NMDA-induced $\left[{ }^{3} \mathrm{H}\right]$ acetylcholine release from brain slices of senescence-accelerated mouse. Int J Dev Neurosci 10, $121-129$.

21. Kawamata T, Akiguchi I, Maeda K, et al. (1998) Age-related changes in the brains of senescence-accelerated mice (SAM): association with glial and endothelial reactions. Microsc Res Tech 43, 59-67.

22. Sureda FX, Gutierrez-Cuesta J, Romeu M, et al. (2006) Changes in oxidative stress parameters and neurodegeneration markers in the brain of the senescence-accelerated mice SAMP-8. Exp Gerontol 41, 360-367.

23. Wu Y, Zhang AQ \& Yew DT (2005) Age related changes of various markers of astrocytes in senescence-accelerated mice hippocampus. Neurochem Int 46, 565-574.

24. Tanaka J, Okuma Y, Tomobe K, et al. (2005) The age-related degeneration of oligodendrocytes in the hippocampus of the senescence-accelerated mouse (SAM) P8: a quantitative immunohistochemical study. Biol Pharm Bull 28, 615-618.

25. Miyazaki H, Okuma Y, Nomura J, et al. (2003) Age-related alterations in the expression of glial cell line-derived neurotrophic factor in the senescence-accelerated mouse brain. J Pharmacol Sci 92, 28-34.

26. Mizushima Y, Kan S, Yoshida S, et al. (2003) Effect of Chotosan, a Kampo medicine, on impairment of passive avoidance performance in senescence accelerated mouse (SAM). Phytother Res 17, 542-545.

27. Rosenzweig ES \& Barnes CA (2003) Impact of aging on hippocampal function: plasticity, network dynamics, and cognition. Prog Neurobiol 69, 143-179.

28. Dahl D, Labkovsky B \& Bignami A (1988) Neurofilament phosphorylation in axons and perikarya: immunofluorescence study of the rat spinal cord and dorsal root ganglia with monoclonal antibodies. J Comp Neurol 271, $445-450$.

29. von Bohlen und Halbach O \& Unsicker K (2002) Morphological alterations in the amygdala and hippocampus of mice during ageing. Eur J Neurosci 16, 2434-2440.

30. Yamamoto T \& Hirayama A (2001) Effects of soft-diet feeding on synaptic density in the hippocampus and parietal cortex of senescence-accelerated mice. Brain Res 902, $255-263$.

31. Smith TD, Adams MM, Gallagher M, et al. (2000) Circuitspecific alterations in hippocampal synaptophysin immunoreactivity predict spatial learning impairment in aged rats. $J$ Neurosci 20, 6587-6593.

32. Yang SZ, Zhang LM, Huang YL, et al. (2003) Distribution of Flk-1 and Flt-1 receptors in neonatal and adult rat brains. Anat Rec A Discov Mol Cell Evol Biol 274, 851-856.

33. Nico B, Mangieri D, Corsi P, et al. (2004) Vascular endothelial growth factor-A, vascular endothelial growth factor receptor-2 and angiopoietin-2 expression in the mouse choroid plexuses. Brain Res 1013, 256-259.

34. Wick A, Wick W, Waltenberger J, et al. (2002) Neuroprotection by hypoxic preconditioning requires sequential activation of vascular endothelial growth factor receptor and Akt. J Neurosci 22, 6401-6407.

35. Matsuzaki H, Tamatani M, Yamaguchi A, et al. (2001) Vascular endothelial growth factor rescues hippocampal neurons from glutamate-induced toxicity: signal transduction cascades. FASEB J 15, 1218-1220.

36. Cao L, Jiao X, Zuzga DS, et al. (2004) VEGF links hippocampal activity with neurogenesis, learning and memory. Nat Genet 36, 827-835.

37. Sun Y, Jin K, Xie L, et al. (2003) VEGF-induced neuroprotection, neurogenesis, and angiogenesis after focal cerebral ischemia. J Clin Invest 111, 1843-1851.

38. Kim BW, Choi M, Kim YS, et al. (2008) Vascular endothelial growth factor (VEGF) signaling regulates hippocampal neurons by elevation of intracellular calcium and activation of calcium/ calmodulin protein kinase II and mammalian target of rapamycin. Cell Signal 20, 714-725.

39. Jin K, Mao XO \& Greenberg DA (2006) Vascular endothelial growth factor stimulates neurite outgrowth from cerebral cortical neurons via Rho kinase signaling. J Neurobiol 66, 236-242.

40. Dalgleish T (2004) The emotional brain. Nat Rev Neurosci 5, 583-589. 
41. Aggleton JP \& Shaw C (1996) Amnesia and recognition memory: a re-analysis of psychometric data. Neuropsychologia 34, 51-62.

42. Brown PD, Davies SL, Speake T, et al. (2004) Molecular mechanisms of cerebrospinal fluid production. Neuroscience $\mathbf{1 2 9}$, 957-970.

43. Emerich DF, Skinner SJ, Borlongan CV, et al. (2005) The choroid plexus in the rise, fall and repair of the brain. Bioessays 27, 262-274.

44. Johanson CE, Duncan JA, Stopa EG, et al. (2005) Enhanced prospects for drug delivery and brain targeting by the choroid plexus-CSF route. Pharm Res 22, 1011-1037.

45. Jakubowska-Doğru E \& Gümüşbaş U (2005) Chronic intracerebroventricular NGF administration improves working memory in young adult memory deficient rats. Neurosci Lett 382, 45-50.
46. Shi L, Linville MC, Tucker EW, et al. (2005) Differential effects of aging and insulin-like growth factor- 1 on synapses in CA1 of rat hippocampus. Cereb Cortex 15, 571-577.

47. de Boer VC, de Goffau MC, Arts IC, et al. (2006) SIRT1 stimulation by polyphenols is affected by their stability and metabolism. Mech Ageing Dev 127, 618-627.

48. Bagchi D, Bagchi M, Stohs SJ, et al. (2000) Free radicals and grape seed proanthocyanidin extract: importance in human health and disease prevention. Toxicology 148, 187-197.

49. Weinreb O, Mandel S, Amit T, et al. (2004) Neurological mechanisms of green tea polyphenols in Alzheimer's and Parkinson's diseases. J Nutr Biochem 15, 506-516.

50. Deprez S, Mila I, Huneau JF, et al. (2001) Transport of proanthocyanidin dimer, trimer, and polymer across monolayers of human intestinal epithelial Caco-2 cells. Antioxid Redox Signal 3, 957-967. 